\title{
2 IN THE HUMAN GASTROINTESTINAL TRACT
}

3

4

5 Tom R. Van de Wiele ${ }^{1 *}$, Agnes G. Oomen ${ }^{2}$, Joanna Wragg ${ }^{3}$, Mark Cave ${ }^{3}$, Mans Minekus ${ }^{4}$,

6 Alfons Hack ${ }^{5}$, Christa Cornelis ${ }^{6}$, Cathy J.M. Rompelberg ${ }^{2}$, Loeckie L. De Zwart ${ }^{2}$, Ben

7 Klinck $^{3}$, Joop Van Wijnen ${ }^{7}$, Willy Verstraete ${ }^{1}$ and Adriënne J.A.M. Sips ${ }^{2}$

8

$9{ }^{1}$ LabMET, Ghent University, Ghent, Belgium

$10{ }^{2}$ National Institute of Public Health and the Environment, Bilthoven, The Netherlands

$11{ }^{3}$ British Geological Survey, Nottingham, United Kingdom

$12{ }^{4}$ TNO Nutrition, Zeist, The Netherlands

$13 \quad{ }^{5}$ Ruhr-Universität Bochum, Bochum, Germany

$14 \quad{ }^{6}$ Vito, Mol, Belgium

$15{ }^{7}$ GG\&GD, Amsterdam, The Netherlands

16

17

18 * Corresponding author. Tom Van de Wiele, LabMET - Ghent university. Coupure Links 
ABSTRACT

This paper presents a multi-laboratory comparison study of in vitro models assessing bioaccessibility of soil-bound lead in the human gastrointestinal tract under simulated fasted

4 and fed conditions. Oral bioavailability data from a previous human in vivo study on the same

5 soil served as a reference point. In general, the bioaccessible lead fraction was significantly $6 \quad(\mathrm{P}<0.05)$ different between the in vitro methods and ranged for the fasted models from $2 \%$ to $733 \%$ and for the fed models from $7 \%$ to $29 \%$. The in vivo bioavailability data from literature were $26.2 \pm 8.1 \%$ for fasted conditions, compared to $2.5 \pm 1.7 \%$ for fed conditions. Under fed conditions, all models returned higher bioaccessibility values than the in vivo bioavailability, whereas three models gave a lower bioaccessibility than bioavailability under fasted conditions. These differences are often due to the method's digestion parameters that need further optimization. An important outcome of this study was the determination that the method for separating the bioaccessible lead from the non-bioaccessible fraction 14 (centrifugation, filtration, dialysis) is crucial for the interpretation of the results. Bioaccessibility values from models that use more stringent separation methods better approximate in vivo bioavailability results, yet at the expense of the level of conservancy. We conclude from this study that more optimization of in vitro digestion models is needed for use in risk assessment. Moreover, attention should be paid to the separation method since it largely influences what fraction of the contaminant is considered bioaccessible.

21 Keywords: Bioaccessibility, bioavailable, intestine, human gut, in vitro digestion, $\mathrm{Pb}$, soil ingestion. 


\section{INTRODUCTION}

2 Lead is a wide spread heavy metal in the environment and can be both acutely and

3 chronically toxic to humans. The nervous system is the most sensitive target of lead exposure

${ }^{[1]}$, however, lead can affect every organ system, leading to anemia, renal problems and

5 hypertension ${ }^{[2]}$. Exposure to lead primarily occurs through inhalation and ingestion of

6 contaminated matrices such as soil. Especially in urban areas, more contamination of soil and

7 dust with lead is reported, due to the higher traffic density, former use of lead based paints,

8 and industrial activities ${ }^{[3]}$. Oral exposure not only to lead, but other environmental

9 contaminants via soil ingestion is an important public health issue. Site-specific risk assessment studies incorporate soil ingestion with a maximal daily intake of 50 and $150 \mathrm{mg}$ soil $\mathrm{d}^{-1}$ for adults and children, respectively ${ }^{[4]}$. Reported human soil intake rates generally range from 1 to $50 \mathrm{mg} / \mathrm{d}$ for adults and from 100 to $500 \mathrm{mg} / \mathrm{d}$ for children ${ }^{[5-7]}$. However, pica afflicted children may show an unusual hand-to-mouth behaviour and can ingest up to $20 \mathrm{~g}$ 14 soil/d ${ }^{[6]}$.

The concepts of bioaccessibility and oral bioavailability are fundamentally important for quantifying the risks that are associated with oral exposure to environmental contaminants. Bioaccessibility refers to the fraction of a contaminant that is released from soil into solution by digestive juices. It represents the maximum amount of contaminant that is available for intestinal absorption. In general, only a fraction of these bioaccessible contaminants can be absorbed by the intestinal epithelium and subsequently transported to the liver via the portal

21 vein for biotransformation. The fraction of parent compound that reaches the systemic 22 circulation is referred to as the bioavailable fraction. Given the fact that bioaccessibility is one of the principal factors limiting the bioavailable fraction, it is an important parameter to assess for risk assessment purposes. 
In current risk assessment practice for the ingestion of soil-bound contaminants, the risk

2 associated to a specific oral dose of a contaminant is compared to toxicological reference 3 values of that contaminant based on intakes from water or food matrices. Soil bound contaminants have however different desorption and complexation processes in the gastrointestinal tract than those ingested with food or water. Calculation of soil contamination related risks based on a similar intake with water or a food matrix may, therefore yield inaccurate bioaccessibility values. An adapted risk assessment method should therefore be developed to assess the specific risks from ingested contaminants that are bound to a matrix such as soil.

Human bioavailability data from actual human feeding tests are scarce ${ }^{[8]}$ and in vivo experiments in general are costly, time consuming and related to important ethical constraints. An alternative is the application of in vitro models that simulate the human gastrointestinal tract. These screening methods are fast, reproducible and can be used to measure the bioaccessible contaminant fraction, as bioaccessibility is an important parameter prior to bioavailability. Several in vitro methods of the human gut have been developed ${ }^{[9-14]}$. Some of these methods have already been applied to measure bioaccessibility of both heavy metals and organic compounds ${ }^{[9,14-16]}$. In a previous paper, we compared five in vitro digestion models that were applied on standard reference soils contaminated with arsenic, cadmium and lead [13]. It was found that the bioaccessible fraction largely depends on the applied in vitro method. If bioaccessibility is to be incorporated in risk assessment procedures for the 21 ingestion of soil contaminants, a better insight in the methodology is warranted.

In this study, the five in vitro digestion models were used to estimate lead bioaccessibility 23 from a Bunker Hill soil. This soil was previously used by Maddaloni et al. ${ }^{[8]}$ to measure oral lead bioavailability in adults. Similar to the in vivo study, we investigated fasted and fed conditions in the gastrointestinal tract. Our intention was not only to evaluate the different in 
1 vitro methods and show their variability with in vivo data as a reference point, but also to

2 highlight areas in which the models should converge to best mimic in vivo results. This 3 comparison study could improve our understanding of how to relate bioaccessibility to oral 4 bioavailability of ingested soil contaminants.

5 


\section{EXPERIMENTAL SECTION}

Soil. The standard reference Bunker Hill soil was kindly provided by Mark Maddaloni. The bioavailability of $\mathrm{Pb}$ in Bunker Hill soil to humans had been determined in an in vivo study by Maddaloni et al. ${ }^{[8]}$.

Experimental design. The bioaccessibility of $\mathrm{Pb}$ in the Bunker Hill soil was assessed with five in vitro digestion models. Four of the models are so called 'static models': the modified PBET method (Physiologically Based Extraction Test) operated by the BGS (United Kingdom); the German E DIN 19738, applied by the Ruhr-Universität Bochum (Germany); the RIVM (the Netherlands) in vitro digestion model and the SHIME procedure used by LabMET (Ghent University, Belgium). The only dynamic gastro-intestinal model used is the TIM method (TNO, the Netherlands). The models are described in more detail below. Maddaloni et al. (1998) investigated two scenarios in their bioavailability study of a lead contaminated soil: fasted conditions in which soil was ingested with water upon overnight fasting and fed conditions where soil was ingested with a standard breakfast meal. In this study, fasted and fed conditions were applied for every in vitro model. However, the composition of the nutrition was in most cases not the same as that used in the in vivo study, since the intrinsic intentions and concepts of the methods (for example simulation of the child's gut) were left unchanged.

$\mathbf{P b}$ analysis. $\mathrm{Pb}$ analysis of the digestive juices, the pellets and the Bunker Hill soil was performed by the Flemish Institute for Technological Research (VITO, Belgium). This way, possible differences in bioaccessibility values that would originate from the different analytical methodology to measure the lead content in the samples were eliminated. The Bunker Hill soil was analyzed in triplicate for lead content using a closed microwave oven 
1 destruction with $\mathrm{HCl} / \mathrm{HNO}_{3}(3+1)$ and ICP-AES quantification. The pellets from the in vitro

2 bioaccessibility tests were dried until constant weight; lead determination was performed the

3 same way as for the soil analyses. Liquid samples were digested by semi-open microwave

4 oven destruction with $\mathrm{HCl} / \mathrm{HNO}_{3}(3+1)$ and ICP-MS or ICP-AES determination depending on

5 the lead concentration in solution. Blank digestion solutions were analyzed as a control.

6

\section{Description of in vitro digestion models.}

PBET (United Kingdom) ${ }^{[14]}$. First $1 \mathrm{~g}$ of soil was weighed into wide mouthed HDPE (high density polyethylene) bottles. $100 \mathrm{~mL}$ of simulated gastric solution (1.25 g pepsin, 0.50 g sodium malate, $0.50 \mathrm{~g}$ sodium citrate, $420 \mu \mathrm{l}$ lactate and $500 \mu \mathrm{l}$ acetate per liter de-ionised water, adjusted to $\mathrm{pH} 2.5$ with concentrated hydrochloric acid) was added to each bottle. The bottles were placed in a water rotator set at $37^{\circ} \mathrm{C}$. For the fed and fasted experiments, $1 \mathrm{~g}$ of Bunker Hill soil was extracted in triplicate for each method. After one hour at $37^{\circ} \mathrm{C}$, a $5.0 \mathrm{~mL}$ aliquot was removed and filtered through a $0.45 \mu \mathrm{m}$ cellulose filter disk for analysis. This extraction sample is known as the stomach phase. Five $\mathrm{mL}$ of the original gastric solution was then back-flushed through the filter into the HDPE bottle to retain the original L/S ratio (liquid (mL) to soil (g) ratio). The conditions in the vessel were then altered from stomach to small intestinal conditions by titration to $\mathrm{pH} 7.0$ with saturated sodium bicarbonate and the addition of $175 \mathrm{mg}$ bile salts and $50 \mathrm{mg}$ pancreatin. The samples were then incubated in the water bath for four hours. These samples represented the small intestine. The experiments to simulate fed state included the addition of $1.0 \mathrm{~g}$ of baby whole milk powder (Cow \& Gate, UK) to the digestive suspension.

Method E DIN 19738 (Germany). The German method E DIN 19738 has its origin in the in vitro digestion models of Rotard et al. ${ }^{[17]}$ and of Hack and Selenka ${ }^{[9]}$. It is a static 
1 gastrointestinal model that uses synthetic digestive juices. Since it is assumed that saliva has a

2 negligible effect on the level of mobilization of contaminants from soil, only synthetic gastric

3 juice, and synthetic intestinal juice was used in the present round robin. Whole milk powder

$4 \quad(50 \mathrm{~g} / \mathrm{L})$ was added to the gastric juice to simulate the influence of food on the mobilization of 5 contaminants. Two g of contaminated dry soil were suspended in $100 \mathrm{~mL}$ gastric juice 6 (diluted $\mathrm{HCl}$ ) for two hours at $\mathrm{pH}$ 2.0. This was followed by the addition of $100 \mathrm{~mL}$ of 7 intestinal juice, the $\mathrm{pH}$ was set to 7.5 using a phosphate buffer, and digestion proceeded for six hours. The temperature was controlled by means of a water bath $\left(37^{\circ} \mathrm{C}\right)$. Mixing occurred with an agitator at $200 \mathrm{rpm}$. The digestion mixture was centrifuged for ten minutes at $7000 \mathrm{~g}$, after which the supernatant was decanted. The residual pellet was stirred in $30 \mathrm{~mL}$ of distilled water for $0.2 \mathrm{~h}$, centrifuged, and the supernatant decanted. The decanted intestinal solutions were combined for analysis.

RIVM (The Netherlands). The fasting model and the composition of its digestive juices have been described in detail by Oomen et al. ${ }^{[18]}$. Briefly, the digestion was started by addition of $9.0 \mathrm{~mL}$ saliva of $\mathrm{pH} 6.5$ to $0.6 \mathrm{~g}$ dry matter soil. This mixture was rotated endover-end at $55 \mathrm{rpm}$ at $37^{\circ} \mathrm{C}$. Then, $13.5 \mathrm{~mL}$ gastric juice of $\mathrm{pH} 1.07$ was added, and rotated at $37^{\circ} \mathrm{C}$. After two hours, $27 \mathrm{~mL}$ duodenal juice $(\mathrm{pH} \mathrm{7.8)}$ and $9 \mathrm{~mL}$ bile juice $(\mathrm{pH} 8.0)$ were added. This mixture was rotated at $37^{\circ} \mathrm{C}$ for 2 hours and subsequently centrifuged at $3000 \mathrm{~g}$ for 5 minutes. The supernatant (total volume $58.5 \mathrm{~mL}$ ) represented the chyme. For the fed model, $6 \mathrm{~mL}$ of simulated saliva (pH 6.8), $4.5 \mathrm{~g}$ of infant formula (macaroni based), and 12 $\mathrm{mL}$ of stimulated gastric juice $(\mathrm{pH} 1.30)$ were added to $0.4 \mathrm{~g}$ dry matter soil. The mixture was rotated end-over-end at about $55 \mathrm{rpm}$ at $37{ }^{\circ} \mathrm{C}$ for $2 \mathrm{~h}$. Subsequently, $12 \mathrm{~mL}$ of stimulated duodenal juice ( $\mathrm{pH} 8.1), 6 \mathrm{~mL}$ of stimulated bile juice $(\mathrm{pH} 8.2)$ and $2 \mathrm{~mL} \mathrm{NaHCO}_{3}(85 \mathrm{~g} / \mathrm{l})$ 
1 chyme was separated from the pellet by centrifugation at $3000 \mathrm{~g}$. For both models, the $\mathrm{pH}$ was

2 determined at the end of the stomach and of the intestinal phase. The composition of the non-

3 stimulated digestive juices is based on human physiology and is described in more detail ${ }^{[18]}$.

4 Stimulated saliva contains more bicarbonate, $\alpha$-amylase and less mucine than non-stimulated

5 saliva. Stimulated gastric juice contains more pepsine, whereas stimulated duodenal and bile

6 juice contains more pancreatine and lipase and five times more bile than non-stimulated 7 duodenal juice.

SHIME (Belgium). The SHIME (Simulator of the Human Intestinal Microbial Ecosystem) procedure as employed in the present set-up represented a static gastrointestinal system simulating the gut of young children. The different digestive juices were added in batch to the same compartment. If needed, the system can be extended to more compartments with larger volumes and $\mathrm{pH}$ gradients. The SHIME in its full configuration includes 3 colon compartments with a mixed microbial community. This was not applied in this study.

Five grams of soil were introduced into $50 \mathrm{~mL}$ of SHIME nutritional medium, which had a starting $\mathrm{pH}$ of 5.2. The gastric $\mathrm{pH}$ was brought to 2.0 for fasted conditions and to 4.0 for fed conditions. SHIME nutritional medium contains per liter sterile distilled water $15 \mathrm{~g}$ Nutrilon plus, $16 \mathrm{~g}$ pectin, $8 \mathrm{~g}$ mucin, $5 \mathrm{~g}$ starch, $1 \mathrm{~g}$ cellobiose, $1 \mathrm{~g}$ glucose and $2 \mathrm{~g}$ proteose peptone. Nutrilon is nutrition for children between 4 and 18 months, and is obtained from Nutricia (Bornem, Belgium). Main constituents are lactose (56\%), fat (12\%), and casein (10\%). After

213 hours of incubation at $37^{\circ} \mathrm{C}, 25 \mathrm{~mL}$ of a solution of pancreatic enzymes and bile salts was supplemented in order to obtain small intestinal fluid. This solution consists of $12 \mathrm{~g} \mathrm{NaHCO}_{3}$, $4 \mathrm{~g}$ bovine bile and $0.9 \mathrm{~g}$ pancreatine per liter of distilled water. This small intestinal suspension had $\mathrm{pH} 6.5$, and was stirred at $150 \mathrm{rpm}$ at $37{ }^{\circ} \mathrm{C}$ for 5 hours. Subsequently, the 
analyzed. When fed conditions were simulated, $200 \mathrm{~mL}$ of gastric juice were suplemented to $5 \mathrm{~g}$ of soil. For the small intestinal digestion, $100 \mathrm{~mL}$ of the enzymatic and bile solution were dosed to the gastric suspension.

TIM (The Netherlands). The TNO intestinal model is a dynamic model that simulates the $\mathrm{pH}$ profile as well as continuous addition of enzymes, bile, and other components during gradual transit of soil through the different compartments of the gastro-intestinal tract ${ }^{[11]}$. The amount of soil and the meal was based on the study presented by Maddaloni et al. ${ }^{[8]}$. One hundred milligrams of soil were introduced in the model with $240 \mathrm{~mL}$ of water or the standard breakfast from the in vivo study to study the fasted and fed situation, respectively. Briefly, the main constituents of the standard breakfast were $25 \mathrm{~g}$ of wheat cereal, $130 \mathrm{~g}$ of whole milk, 1 large hard-boiled egg (approximately $48 \mathrm{~g}$ ), $50 \mathrm{~g}$ of firm whole wheat bread, $6 \mathrm{~g}$ of butter, $5 \mathrm{~g}$ of jam/preservatives, and $6 \mathrm{~g}$ of white sugar. The halftime of gastric emptying was $30 \mathrm{~min}$ for the fasted and 80 min for the fed situation.

The initial gastric $\mathrm{pH}$ during fed situation was 5 and gradually decreased to $3.5,2.5$ and 2 after 30, 60 and 90 minutes, respectively. During the fasted situation, the gastric $\mathrm{pH}$ started at $\mathrm{pH} 4.5$ whereafter it decreased to $3.2,2.8$ and 1.8 in 10, 20 and 40 minutes, respectively. Subsequently, the soil was gradually transferred to the intestinal compartments, representing the duodenum ( $\mathrm{pH}$ 6.5), the jejunum $(\mathrm{pH}$ 6.8) and the ileum ( $\mathrm{pH}$ 7.2). The gastric and duodenal secretion was set to 0.5 and $1 \mathrm{~mL} /$ minute, respectively. The total digestion time is 360 minutes. The chyme is mixed and transported by peristaltic movements. Dialysis membranes (Hospal, MWcutoff 5000-10000) are used to remove bioaccessible contaminants, digestive metabolites and water from the chyme.

\section{Round robin}


Experimental design. The bioaccessibility of $\mathrm{Pb}$ in the Bunker Hill soil was assessed with

2 the in vitro digestion models described above. The soil was distributed by the RIVM institute,

3 which ensured that the input material in the different digestion models was the same. Each of

4 the institutes applied their in vitro model on the soil, both with fasted conditions as with fed

5 conditions. The PBET and RIVM models measured lead bioaccessibility in both the stomach

6 compartment alone as the stomach/intestine compartments combined. The RIVM method also

7 performed digestion experiments at 2 different L/S ratios, 100 and 1000. Bioaccessibility was

8 calculated as:

9 Bioaccessibility $(\%)=100 \% *$ contaminant mobilized from soil during digestion $(\mu) /$

10 contaminant present in soil before digestion $(\mu)$

11 Contaminant concentrations in the chyme and pellet were determined. This allowed a mass

12 balance for each of the methods to be calculated. It should be noted that the respective in vitro models applied different fed conditions according to their original experimental setup. Thus, the fed conditions were not standardized across all methods in this study, which will add up to large the variability that exists between the different models.

Statistical information. Standard practice for comparing different tests is to use analysis of variance (ANOVA). However, since there are relatively few data points for the methods used in this study, there are different numbers of replicate measurements for each test and there is no guarantee of the data being normally distributed, a simpler approach to a statistical 21 comparison of these tests was to use a permutation test ${ }^{[19]}$. In this instance the null hypothesis is that there is no significant difference in the bioaccessibility of lead as measured by the different methods. In order to test this, the individual mean values for each method are calculated along with the overall mean of all the tests being considered. The absolute differences between each test mean value and the overall mean are then calculated and 
1 summed. This provides the benchmark statistic. The data for all the tests are then randomly

2 shuffled (retaining the same number of data points for each method) and the test statistic is

3 recalculated. This process of random shuffling and calculation of the test statistic is repeated

$4 \quad 10,000$ times recording the test statistic each time.

5 The number of times the test statistic exceeds the benchmark statistic is recorded. If there

6 is no significant difference between the methods then test statistic is likely to exceed

7 benchmark statistic in a large proportion of the reshuffling trials. If, however, there is a

8 significant difference between the methods then it is unlikely that a randomly shuffled trial

9 will exceed the benchmark statistic. The probability cut-off chosen in this study is that of 5\%

10 (i.e. $\mathrm{p}=0.05$ ). Therefore if $5 \%$ or more of the trials are greater than the benchmark statistic

11 then the null hypothesis is confirmed and there is no significant difference between the

12 methods. If there are less than $5 \%$ of the reshuffled trials greater than the test statistic then the

13 results of the different methods are significantly different. The advantage of using this

14 approach is that it is simple to carry out (calculations were carried out in Excel using

15 Resampling Stats software ${ }^{[19]}$ ) and no assumptions about the distributions of the data need to

16 be made. 
RESULTS

Our quality assurance and quality control steps found that the lead concentration in the Bunker Hill soil was $3060 \pm 55 \mathrm{mg} \mathrm{Pb} / \mathrm{kg}$ soil similar to the value of $2924 \pm 36 \mathrm{mg} \mathrm{Pb} / \mathrm{kg}$ soil types ranged from at least $92 \%$ up to $98 \%$. The fed digestion types delivered lower recovery data ranging from $73 \%$ to $93 \%$ (Table 1). It should be noted that the lead analysis was performed by one analytical laboratory with all participants sending their samples to this institute for analysis.

All bioaccessibility data for the different models are summarized in Table 1. For the fed status, all models except TIM gave bioaccessibility values that were significantly higher than the in vivo bioavailability. For the fasted status, the PBET, DIN and SHIME methods underestimated bioavailability, whereas the RIVM method was higher. Only the TIM model generated bioaccessibility results that were not significantly different from the in vivo bioavailability data, both under fed and fasted conditions.

Bioaccessibility data from the different in vitro methods were largely dependent on the absence or presence of food components. Figure 1 shows a comparison of the differences between the average bioaccessibility for the fasted and fed conditions. The resampling test showed that, apart for the RIVM stomach extraction for both $1 / 100$ and $1 / 1000$ liquid to solid ratios, these differences are significant. In analogy with the human bioavailability data, the TIM model showed much lower bioaccessibility results when fed conditions were simulated. The same trend was observed for RIVM intestine 1/100 and RIVM intestine $1 / 1000$, but to a lesser extent. Higher bioaccessibility data for fed conditions were obtained with the PBET intestine method, the SHIME and DIN method. The presence of food components in the digest suspensions thus plays a major role for the outcome of the bioaccessibility results. Interestingly, the bioaccessibility results obtained with the dynamic TIM model were not 
1 significantly different from the in vivo bioavailability data, both under fasted and fed 2 conditions.

3

4 Table 1. Lead recovery percentage, liquid to soil ratio and bioaccessibility results ( \pm standard deviation) for the

5 different in vitro gastrointestinal digestions of Bunker Hill Soil (2924 $\pm 36 \mathrm{mg} \mathrm{Pb} / \mathrm{kg}$ soil DW)

\begin{tabular}{|c|c|c|c|c|}
\hline & $\mathbf{n}$ & \% recovery & $\mathbf{L} / \mathbf{S}$ & \% Bioaccess. \\
\hline \multicolumn{5}{|l|}{ PBET } \\
\hline Fasted & 3 & 98 & 100 & $13.0 \pm 0.8$ \\
\hline Fed & 2 & 93 & 100 & $21.8 \pm 0.4$ \\
\hline \multicolumn{5}{|l|}{ DIN } \\
\hline Fasted & 3 & 96 & 100 & $13.6 \pm 0.6$ \\
\hline Fed & 3 & 88 & 100 & $28.6 \pm 1.6$ \\
\hline \multicolumn{5}{|l|}{ RIVM } \\
\hline Fasted & 3 & $90 \pm 2$ & 98 & $31.8 \pm 2.5$ \\
\hline Fed & 3 & $78 \pm 8$ & 101 & $23.9 \pm 2.4$ \\
\hline \multicolumn{5}{|l|}{ SHIME } \\
\hline Fasted & 2 & 92 & 15 & $2.0 \pm 0.1$ \\
\hline Fed & 3 & 83 & 62 & $24.1 \pm 0.1$ \\
\hline \multicolumn{5}{|l|}{ TIM } \\
\hline Fasted & 2 & & 51 & $32.5 \pm 4.5$ \\
\hline Fed & 2 & & 51 & $7.0 \pm 1.5$ \\
\hline
\end{tabular}

6

7

8 


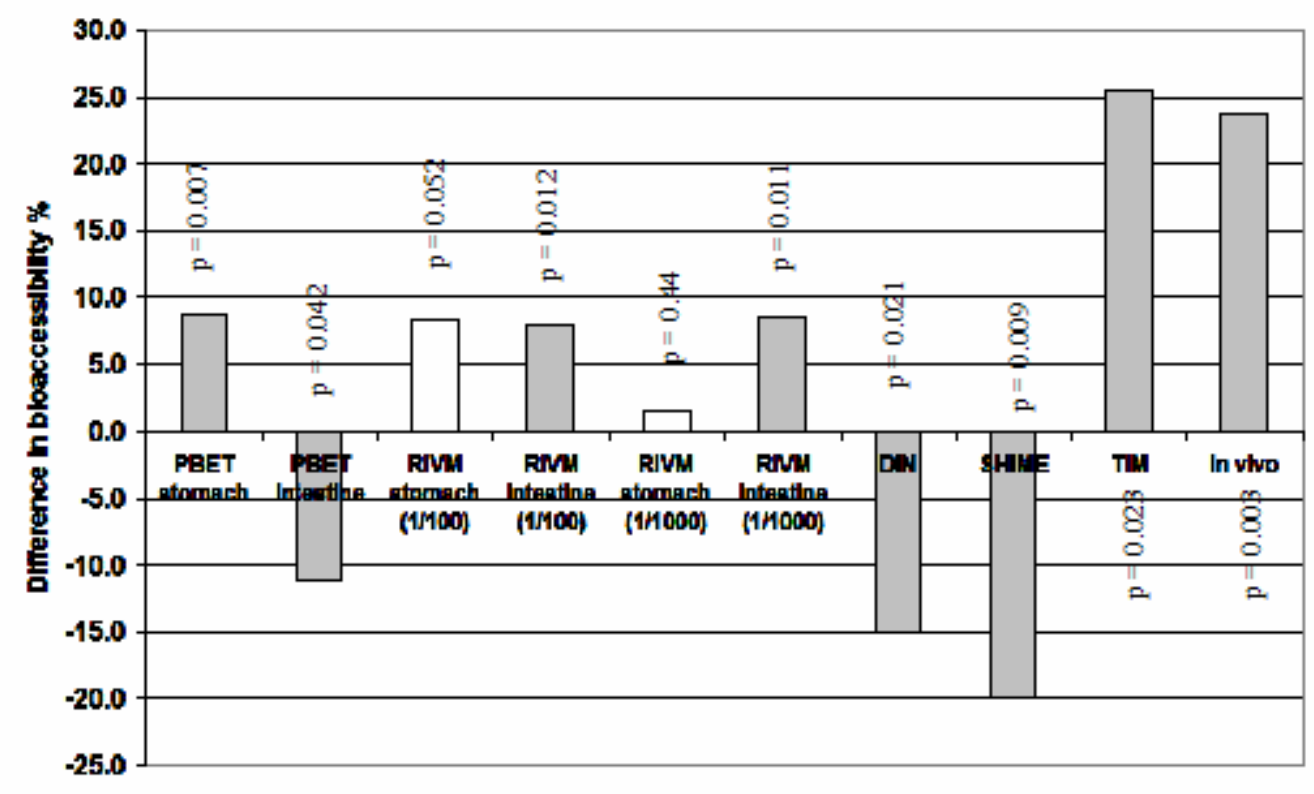

Figure 1. Difference in Bioaccessibility/Bioavailability between fasted and fed condtions ( $p$ values $<0.05$ indicate a significant difference between fed and fasted). Grey bars are significant, white bars are not significant. A positive value means that bioaccessibility was higher under fasting conditions than under fed conditions.

Fasted conditions. The in vitro methods differed significantly from each other and the in vivo data ( $\mathrm{p}=0.003$ ) (Figure 2). The SHIME method showed the lowest bioaccessibility value (2.0\%), followed by PBET intestine (13.0\%) and DIN (13.6\%), whereas the RIVM intestine method (31.8\%) and TIM (32.5\%) displayed higher bioaccessibility values (Table 1), quite comparable with the oral bioavailabitiliy value of $26.2 \%$. Lead bioaccessibility values from the PBET (25.0\%) and RIVM (70.9\%) stomach digests were higher than the respective small intestine and stomach digests combined. RIVM also performed digestions at two different liquid to soil (L/S) ratios, being approximately 1000:1 and 100:1 (Figure 2). L/S ratios of 1000 caused higher bioaccessibilities, $85.9 \%$ and $47.4 \%$ for stomach and small intestine respectively, compared to digests at a L/S ratio of $100,70.9 \%$ and $31.8 \%$ for stomach and small intestine, respectively. 


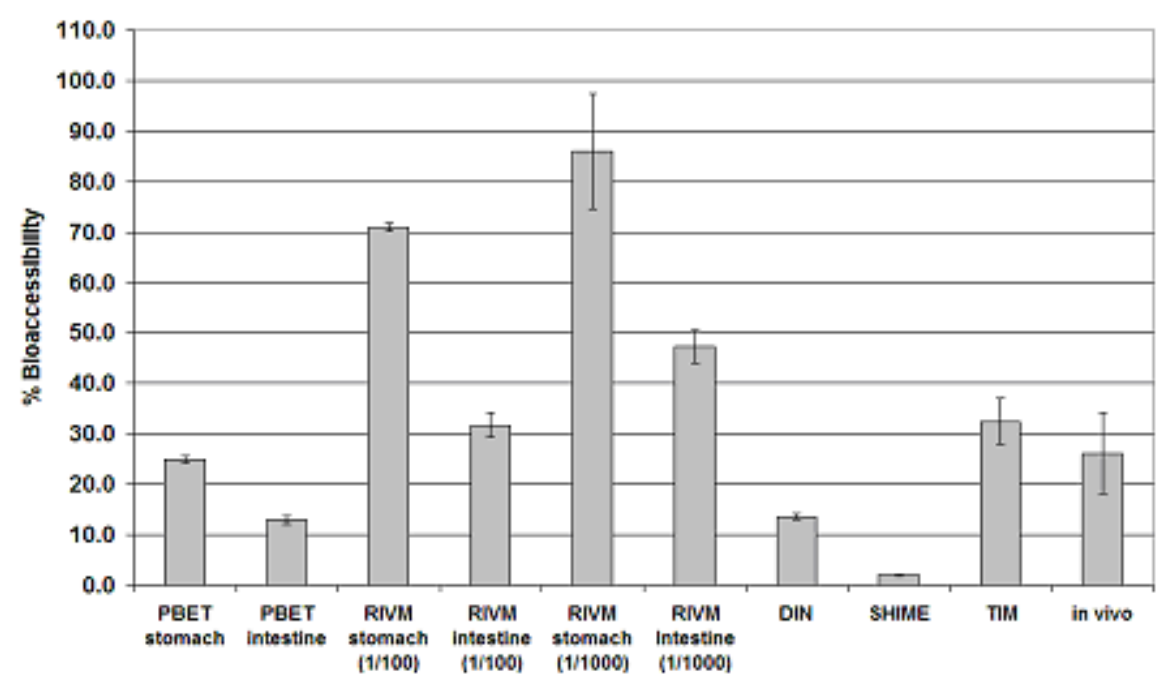

2 Figure 2. Comparison between methods of the bioaccessibility/bioavailability data upon simulated fasted

3 conditions. Bars show the range of results that were obtained with different replicates within one method.

Fed conditions. Significant differences $(\mathrm{p}=0.0008)$ between all methods, including the in 5 vivo study by Maddaloni et al. (1998) ${ }^{[8]}$, were obtained. The bioaccessibility values from all 6 small intestinal digestions were higher than the in vivo bioavailability results (Figure 3). The 7 DIN method had the highest bioaccessibility value (28.6\%), whereas the digestion models SHIME, RIVM and PBET produced slightly lower values of $24.1 \%, 23.9 \%$ and $21.8 \%$, respectively. These bioaccessibility results were significantly different from the bioavailability data. The TIM method on the contrary returned the lowest fed bioaccessibility 11 value, $7.0 \%$, quite comparable with the in vivo bioavailability of $2.5 \%$. The stomach 12 bioaccessibility results for the RIVM method at L/S ratios of $100(62.5 \%)$ and $1000(84.4 \%)$ were higher than the small intestine bioaccessibilities (23.9\% and $38.8 \%$ respectively). The PBET method simulating fed conditions returned a lower bioaccessibility value in the stomach $(16.2 \%)$ than in the small intestinal digest $(21.8 \%)$, this in contrast to the fasted conditions of the PBET method in which the opposite was observed. 


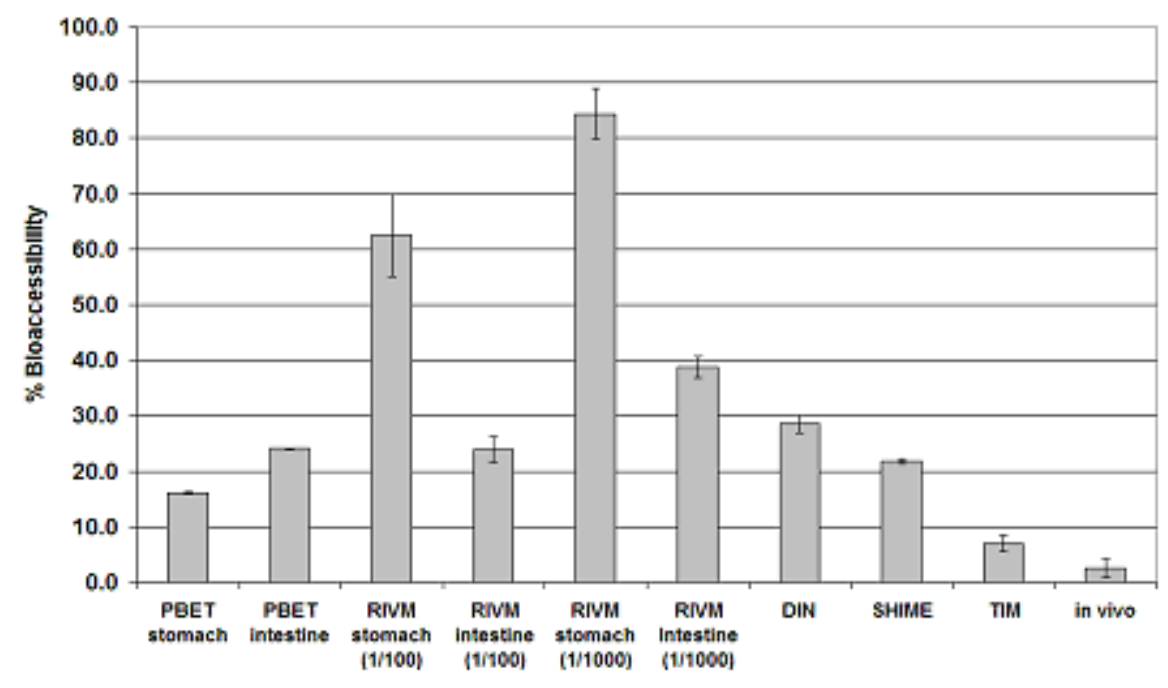

2 Figure 3. Comparison between methods of the bioaccessibility/bioavailability data upon simulated fed

3 conditions. Bars show the range of results that were obtained with different replicates within one method.

Bioaccessibility separation method. For each in vitro model a different separation method was applied to separate the bioaccessible from the non-bioaccessible fraction: $3000 \mathrm{~g}$ centrifugation (RIVM method), $7000 \mathrm{~g}$ centrifugation (SHIME and DIN), microfiltration (PBET) and dialysis (TIM). As the separation method will largely influence which fraction of the solubilized lead is considered as bioaccessible, the RIVM performed additional tests on the Bunker Hill soil under fed conditions with the same breakfast formulation as the one used

11 in the in vivo study from Maddaloni et al. (1998) ${ }^{[8]}$ and that used with the TIM model. As expected, it was found that the bioaccessibility was largely influenced by the separation

13 method with $3000 \mathrm{~g}$ centrifugation yielding the highest bioaccessibility value (31.5\%), 14 followed by microfiltration (22\%) and dialysis (3.5\%) (Table 2). the separation method

\begin{tabular}{cc}
\hline Separation method & Bioaccessibility (\%) \\
\hline Centrifugation $(3000 \times g)$ & 31.5 \\
Microfiltration $(0.45 \mu \mathrm{m})$ & 22 \\
Ultrafiltration $(5 \mathrm{kDa})$ & 3.5 \\
\hline
\end{tabular}




\section{DISCUSSION}

Oral bioavailability is a crucial parameter to incorporate when assessing the risks from the oral uptake of contaminated matrices. Since in vivo methods are slow, costly and complex to measure bioavailability, in vitro methods of the human gut offer a fast and reproducible methodology to be used in risk assessment. Yet, these methods provide bioaccessibility factors defined as the contaminant fraction which solubilizes from its matrix during gastrointestinal digestion and which becomes available for intestinal absorption. Therefore, bioaccessibility data should always return higher values than bioavailability data.

In this study, all in vitro methods yielded bioaccessibility values under fed conditions that were higher than the bioavailability value from the in vivo reference point ${ }^{[8]}$. These methods could therefore serve as conservative tools to estimate the oral bioavailability. Yet it should be noted that the level of conservancy for some models is so high that their practical use for risk assessment purposes can be questioned. Under fasted conditions, however, the PBET, DIN and SHIME in vitro methods yielded lower bioaccessibility values than the in vivo bioavailability reference point. The digestion parameters that were applied in these methods for this study therefore need optimization before the methods become applicable to assess bioaccessibility and use in risk assessment.

Interestingly, the same in vitro methods - DIN, PBET and SHIME - gave higher bioaccessibility values during fed conditions than during fasted conditions, whereas the RIVM and TIM-model and the in vivo reference point showed the reverse trend. This difference can mainly be attributed to the fact that the food composition from the fed models was not standardized. DIN, PBET and SHIME applied fed conditions from previous experimental setups, primarily based on formulations with whole milk powder. In contrast, RIVM and TIM originally did not have a standardized fed model for their in vitro method. RIVM therefore used a macaroni based infant formula whereas the TIM model applied the 
1 standardized breakfast meal as Maddaloni et al., ${ }^{[8]}$ who also observed that lead bioavailability

2 decreased upon soil ingestion under fed conditions. Future experiments would certainly need

3 standardized fed conditions in order to compare bioaccessibility values from different in vitro

4 methods. Additionally, when incorporating fed conditions for bioaccessibility measurements,

5 it is also necessary to increase the enzyme and bile concentrations, this to correspond to the in

6 vivo higher enzyme and biliary secretion rates during food digestion. Currently, most in vitro

7 models do not apply higher enzyme and bile concentrations under fed conditions.

The lower lead bioaccessibility in the presence of a food matrix, as observed by the RIVM and TIM models, may be attributed to lead complexation. In a previous study, a decrease in intestinal lead absorption was noted when released lead was complexed to 11 rhamnogalacturonan-II dimers, a pectide polysaccharide of the cell wall of fruits and vegetables ${ }^{[20]}$. The presence of nutrition in the gastrointestinal juice means that more dissolved organic matter is present, providing more complexation niches for lead in solution. However, this also means that lead is in a complexed state with solubilized organic material, rather than in a freely absorbable state. This will be reflected in a lower fraction of released lead that can actually be considered for small intestinal absorption in vivo. Obviously, intestinal absorption is not considered by any of the in vitro methods, but preceeding steps to intestinal absorption, such as luminal solubilization and complexation processes, are considered when determining the bioaccessible lead fraction.

There are several explanations why 3 of the 5 in vitro methods return lower 21 bioaccessibility data under fasted conditions than the in vivo bioavailability reference value. 22 Firstly, there are several digestion parameters which need further optimization. The applied liquid to soil ratio (L/S) of 15 in the SHIME method for example is very low compared to the $\mathrm{L} / \mathrm{S}$ ratios of around 100 in the other models. It is thus much more difficult for lead to enter the solubilized phase under low L/S conditions than high L/S conditions. Other digestion 
1 parameters such as stomach $\mathrm{pH}$, residence time and bile salt concentrations also affect the

2 outcome of a bioaccessibility measurement ${ }^{[9,21]}$. However, it is much too complicated to draw

3 concise conclusions out of the limited number of data from this study. Several methods are

4 commonly used to simulate a child's gastrointestinal tract. They would therefore need further

5 optimization and standardization to more accurately simulate the digestive conditions that

6 occur in the adult gastrointestinal tract.

7 However, there is one important factor which needs further attention: the bioaccessibility separation method. Bioaccessibility describes the fraction of the chemical that desorbs from

9 the soil matrix and is available for intestinal absorption ${ }^{[22]}$. Although this definition is generally agreed upon, it is remarkable to see that there are so many approaches to separate the bioaccessible fraction from the non-bioaccessible fraction: $3000 \times \mathrm{g}, 3500 \times \mathrm{g}$ or $7000 \times \mathrm{g}$ centrifugation, $0.45 \mu \mathrm{m}$ filtration, $5 \mathrm{kDa}$ ultrafiltration ${ }^{[9,13,14,23,24]}$. To infer which method is best to measure the bioaccessible fraction, we should have a clear understanding of the relationship between bioaccessibility and oral bioavailability.

As explained by Oomen et al., ${ }^{[13]}$, the oral bioavailability of a chemical depends on three crucial steps: 1) bioaccessibility, 2) intestinal absorption and 3) metabolism by human biotransformation enzymes. Oral bioavailability is therefore calculated as:

$\mathrm{F}=\mathrm{F}_{\mathrm{BAcc}} * \mathrm{~F}_{\mathrm{Abs}} * \mathrm{~F}_{\mathrm{Met}}$

with $\mathrm{F}$ the bioavailable fraction, $\mathrm{F}_{\mathrm{BAcc}}$ being the bioaccessible fraction, $\mathrm{F}_{\mathrm{Abs}}$ being the fraction absorbed and $\mathrm{F}_{\text {Met }}$ being the fraction which escapes human metabolism. It should be noted that enterocyte and liver metabolites from the intestinally absorbed contaminant are not taken up in this definition of oral bioaccessibility. This definition only refers to the parent compound that reaches systemic circulation. To accurately predict oral bioavailability, it should be assessed what the sensitivity is of these 3 factors to matrix effects. Clearly, the metabolism factor $F_{\text {Met }}$ is insensitive to luminal matrix effects, whereas the bioaccessible 
1 fraction $\mathrm{F}_{\mathrm{BAcc}}$ is highly sensitive since matrix effects determine the luminal processes of

2 mobilization, complexation, desorption. Estimation of the bioaccessible fraction in the gut

3 therefore requires a separation step that only considers those compounds in the intestinal

4 suspension that come into consideration for intestinal transport.

The importance of the separation method was clearly illustrated in this study by the RIVM

6 method which has applied the three main separation methods for measuring the bioaccessible

7 lead fraction of the Bunker Hill soil upon digestion under fed conditions (Table 2). From

8 these data and the previous discussion, it can be concluded that the separation method is

9 crucial when interpreting bioaccessibility results. This also has consequences when

comparing lead bioaccessibility to oral bioavailability data using the above equation. For lead as a heavy metal, liver metabolism is not relevant, so a $\mathrm{F}_{\text {Met }}$ value of $100 \%$ is assumed. The intestinal transport of lead is inherent to the intestinal epithelium and is a constant, hence $\mathrm{F}_{\mathrm{Abs}}$ is a constant. Since the separation method is not part of the digestion process, the bioaccessible fraction $\mathrm{F}_{\mathrm{Bacc}}$ should also be a constant under standardized digestion conditions. Assuming that dialysis is the closest approach to bioaccessibility, $\mathrm{F}_{\mathrm{BAcc}}$ is $3.5 \%$ and $7 \%$ for the RIVM and TIM method, respectively. The other (milder) separation methods generate higher bioaccessibility values that may overestimate the actual bioaccessible fraction. This adds to the uncertainty of the risk assessment process. However, it should be kept in mind that using more stringent separation methods such as ultrafiltration will also decrease the level of conservancy, which is something that needs careful consideration, especially for human health risk assessment where safety factors must always be included.

In summary, the ultrafiltration separation method offers the most accurate method to measure the bioaccessible fraction of a contaminant in the human gut and closely approaches the oral bioavailability. In their current configuration, both the RIVM and TIM model give a conservative estimate of bioavailability under fasted aswell as fed conditions, with TIM 
1 approaching the bioavailability value the most due to the stringent ultrafiltration step. In

2 addition, there are still many differences in digestion characteristics between in vitro methods

3 of the gastrointestinal tract. The BioAccessibility Research Group of Europe (BARGE)

4 currently develops a batch based Barge-unified method that focuses on both digestion

5 characteristics as separation methods. Additionally, as the presence of food components

6 significantly affects the bioavailability process, standardized fed conditions will be developed

7 as well. This method will give additional recommendations to researcher and risk assessors

8 that want to develop a protocol to test the bioaccessible fraction of ingested soil contaminants.

9 The intent is to provide a standardized method that will provide a conservative estimate of the 10 oral bioavailable fraction of contaminants from soil to be used in human health risk 11 assessment.

12

\section{ACKNOWLEDGEMENTS}

The authors would like to thank Mark Maddaloni for kindly providing the Bunker Hill soil. Tom Va de Wiele is a Post Doctoral Fellow of the Fund for Scientific Research - Flanders (Belgium).

REFERENCES

Rosen, J. F., Toxicology 1995, 97(1-3), 11-17.

ATSDR. "Case studies in environmental medicine: Lead toxicity ". Agency for Toxic Substance and Disease Registry, US Department of Health and Human Services, Atlanta (2002).

ATSDR. "Toxicological profile for lead". Agency for Toxic Substance and Disease Registry, US Department of Health and Human Services, Atlanta (1999). 
1 EPA, U. "Exposure factors handbook", Vol. 1. US environmental Protection Agency, Washington DC (1997).

Brunekreef, B., Noy, D. and Clausing, P., American Journal of Epidemiology 1987, 125(5), 892-898.

Calabrese, E. J., Stanek, E. J., James, R. C. and Roberts, S. M., Environmental Health Perspectives 1997, 105(12), 1354-1358.

Vanwijnen, J. H., Clausing, P. and Brunekreef, B., Environmental Research 1990, 51(2), 147162.

Maddaloni, M., Lolacono, N., Manton, W., Blum, C., Drexler, J. and Graziano, J., Environmental Health Perspectives 1998, 1061589-1594.

Hack, A. and Selenka, F., Toxicology Letters 1996, 88(1-3), 199-210.

Jin, Z. W., Simkins, S. and Xing, B. S., Environmental Toxicology and Chemistry 1999, 18(12), 2751-2758.

Minekus, M., Marteau, P., Havenaar, R. and Huisintveld, J. H. J., Atla-Alternatives to Laboratory Animals 1995, 23(2), 197-209.

Molly, K., Woestyne, M. V. and Verstraete, W., Applied Microbiology and Biotechnology 1993, 39(2), 254-258.

Oomen, A. G., Hack, A., Minekus, M., Zeijdner, E., Cornelis, C., Schoeters, G., Verstraete, W., Van de Wiele, T., Wragg, J., Rompelberg, C. J. M., Sips, A. and Van Wijnen, J. H., Environmental Science \& Technology 2002, 36(15), 3326-3334.

Ruby, M. V., Davis, A., Schoof, R., Eberle, S. and Sellstone, C. M., Environmental Science \& Technology 1996, 30(2), 422-430.

Oomen, A. G., Sips, A., Groten, J. P., Sijm, D. and Tolls, J., Environmental Science \& Technology 2000, 34(2), 297-303. 
1 Ruby, M. V., Fehling, K. A., Paustenbach, D. J., Landenberger, B. D. and Holsapple, M. P., Environmental Science \& Technology 2002, 36(22), 4905-4911.

Rotard, W., W., C., Knoth, W. and Mailahn, W. "Bestimmung der resroptionsverfügbaren pcdd/pcdf aus kieselrot". UWSF-Z Umweltchem Ökotox (1995), pp. 3-9.

Oomen, A. G., Rompelberg, C. J. M., Bruil, M. A., Dobbe, C. J. G., Pereboom, D. and Sips, A., Archives of Environmental Contamination and Toxicology 2003, 44(3), 281-287.

ResamplingStats. "Resampling stats in excel" Resampling Stats Inc., Arlington (2001).

Tahiri, M., Pellerin, P., Tressol, J. C., Doco, T., Pepin, D., Rayssiguier, Y. and Coudray, C., Journal of Nutrition 2000, 130(2), 249-253.

Hamel, S. C., Buckley, B. and Lioy, P. J., Environmental Science \& Technology 1998, 32(3), $358-362$.

Ruby, M. V., Schoof, R., Brattin, W., Goldade, M., Post, G., Harnois, M., Mosby, D. E., Casteel, S. W., Berti, W., Carpenter, M., Edwards, D., Cragin, D. and Chappell, W.,

Arkbage, K., Verwei, M., Havenaar, R. and Witthoft, C., Journal of Nutrition 2003, 133(11), $3678-3683$.

Waisberg, M., Black, W. D., Waisberg, C. M. and Hale, B., Food and Chemical Toxicology 2004, 42(5), 835-842.

Weber, L. P. and Lanno, R. P., Environmental Toxicology and Chemistry 2001, 20(5), 1117 1124. 